\title{
Acta Pediátrica de México, una nueva etapa respaldada en una sólida trayectoria
}

\author{
Acta Pediátrica de México, a \\ new era supported by a solid \\ background
}

Historia y tradición

Acta Pediátrica de México es una de las publicaciones científicas con mayor historia, tradición y alcance en el campo de la Pediatría en México y en América Latina.

Desde su inicio ha tenido reconocimiento y seguimiento tanto de lectores como de colaboradores que traspasan fronteras geográficas y culturales. Hoy en día su audiencia sabe que en cada número se ofrece información actual, útil y práctica para el pediatra.

Como publicación oficial del Instituto Nacional de Pediatría, Acta Pediátrica de México no puede conformarse con el éxito y los logros obtenidos al día de hoy; por el contrario, debe aspirar siempre a más y redefinir sus alcances.

Vanguardia y compromiso

El consejo editorial tiene el compromiso de llevar a la revista a la vanguardia y establecer un estandarte de la pediatría hispanoamericana en la literatura médica internacional. Para lograr estos objetivos es indispensable la incorporación y actualización de los procesos editoriales acordes al contexto de la realidad actual.

Durante el año 2014 Acta Pediátrica de México modificó su sistema de sometimiento y revisión de manuscritos a una plataforma electrónica conocida como OJS (Open Journal Systems), dicha plataforma permite realizar la gestión editorial de una manera más profesional, expedita y auditable, además de facilitar el acceso y la difusión del material publicado de una manera automatizada.

También se creó la página oficial, www.actapediatrica.org.mx, que fungirá como la imagen de Acta Pediátrica de México hacia sus lectores y el mundo. En el portal se cuenta con el contenido actualizado de la revista, un canal de noticias y un área de registro e información para los colaboradores. Así mismo, esta página funciona como una herramienta generadora de recursos para hacer de la revista un proyecto sustentable y siempre dispuesto al mejoramiento continuo.

De un sendero a una autopista

La generación, publicación y difusión de conocimientos ha crecido exponencialmente y hoy en 
día es imposible mantenerse actualizado a tal ritmo. La disponibilidad de recursos informáticos y de comunicación han hecho una realidad lo que hace pocos años Ilamábamos "globalización". Gracias a esta interconectividad inmediata el conocimiento generado y publicado en un sitio particular está disponible, casi simultáneamente, en el resto del mundo. Este escenario, aunque fascinante y de proporciones previamente inimaginables, constituye un reto para clasificar el origen y calidad de la información publicada.

Los índices electrónicos de revistas científicas proporcionan actualmente la estrategia más aceptada para concentrar, clasificar y calificar a este tipo de publicaciones.

Durante 2014 Acta Pediátrica de México fue evaluada y aceptada para ser incluida en Scopus (que es la base de datos más grande de resúmenes y citas de la literatura científica revisada por pares). En este mes Acta Pediátrica de México ha completado la aplicación para su inclusión en el Índice de Revistas Mexicanas de Investigación Científica y Tecnológica del Consejo Nacional de Ciencia y Tecnología (CONACyT); de lograr este objetivo Acta Pediátrica de México se verá beneficiada directamente por el respaldo institucional correspondiente y por la incorporación a la red SciELO (Scientific Electronic Library Online).

En los siguientes meses se completarán las aplicaciones para el índice de la Biblioteca Na- cional de Medicina de los Institutos Nacionales de Salud de Estados Unidos de Norte América (MEDLINE) y del índice DOAJ (Directory of Open Access Journals). Su inclusión en estos dos índices permitirá incrementar sustancialmente la difusión y citación de su contenido, calcular el factor de impacto de la revista e incorporar a Acta Pediátrica de México a la autopista de las publicaciones médicas científicas indexadas.

Agradecimiento a colaboradores

Acta Pediátrica de México es una creación del Instituto Nacional de Pediatría, inicialmente con proyección nacional pero, gracias a la invaluable participación de sus autores y revisores, ha crecido mucho más.

Este 2015 Acta Pediátrica de México fortalecerá la calidad, diversidad y competencia de contenidos, por lo que requiere de la colaboración, soporte y retroalimentación de la comunidad científica y académica para lograr los objetivos aquí planteados. Así, los invitamos a formar parte del equipo de autores, lectores y colaboradores visitando nuestro portal www.actapediatrica.org. mx y a formar parte de nuestras redes sociales en facebook.com/ActaPediatricaMexico y en twitter: @ActaPedMex.

Dr. Armando Partida Gaytán Coeditor Acta Pediátrica de México 